\title{
Влияние влажности окружающей среды на электрическую проводимость полиморфных $\mathrm{Ga}_{2} \mathrm{O}_{3}$-структур
}

\author{
(C) А.В. Алмаев ${ }^{1}$, В.И. Николаев ${ }^{2,3}$, С.И. Степанов ${ }^{2,3}$, Н.Н. Яковлев ${ }^{1, \uparrow, ~ А . И . ~ П е ч н и к о в ~}{ }^{3}$, \\ Е.В. Черников ${ }^{1}$, Б.О. Кушнарев ${ }^{1}$ \\ ${ }^{1}$ Национальный исследовательский Томский государственный университет, \\ 634050 Томск, Россия \\ ${ }^{2} \mathrm{OOO} \mathrm{„Совершенные} \mathrm{кристаллы“,}$ \\ 194064 Санкт-Петербург, Россия \\ ${ }^{3}$ Физико-технический институт им. А.Ф. Иоффе Российской академии наук, \\ 194021 Санкт-Петербург, Россия \\ ฯ E-mail: nik_mr_x@mail.ru
}

Поступила в Редакцию 27 октября 2020 г.

В окончательной редакции 5 ноября 2020 г.

Принята к публикации 5 ноября 2020 г.

Исследовано влияние влажности окружающей среды на электрическую проводимость структур $\alpha-\mathrm{Ga}_{2} \mathrm{O}_{3}$ и $\alpha-\mathrm{Ga}_{2} \mathrm{O}_{3} / \varepsilon-\mathrm{Ga}_{2} \mathrm{O}_{3}$. Полиморфные эпитаксиальные слои $\mathrm{Ga}_{2} \mathrm{O}_{3}$ осаждались методом хлоридной газофазной эпитаксии на сапфировые подложки. В качества контактов использовались $\mathrm{Pt}$ и $\mathrm{Pt} / \mathrm{Ti}$. Обнаружено, что вольт-амперные характеристики структур $\mathrm{Pt} / \alpha-\mathrm{Ga}_{2} \mathrm{O}_{3} / \mathrm{Pt}$ и $\mathrm{Pt} / \mathrm{Ti} / \alpha-\mathrm{Ga}_{2} \mathrm{O}_{3} / \varepsilon-\mathrm{Ga}_{2} \mathrm{O}_{3} / \mathrm{Ti} / \mathrm{Pt}$ имеют высокую чувствительность к влажности атмосферы в области температур $25-100^{\circ} \mathrm{C}$. Установлено, что влияние водяных паров на вольт-амперные характеристики является обратимым и наиболее существенные изменения тока в образцах наблюдаются при относительной влажности $\mathrm{RH} \geq 60 \%$. C повышением температуры эффект влияния влажности атмосферы на вольт-амперные характеристики уменьшается и при температурах $T>100^{\circ} \mathrm{C}$ пропадает. Полученные экспериментальные результаты объясняются в рамках механизма Гротгусса.

Ключевые слова: $\alpha-\mathrm{Ga}_{2} \mathrm{O}_{3}, \varepsilon-\mathrm{Ga}_{2} \mathrm{O}_{3}$, полиморфные эпитаксиальные пленки, хлоридная газофазная эпитаксия, вольт-амперные характеристики, влажность атмосферы.

DOI: 10.21883/FTP.2021.03.50606.9546

\section{1. Введение}

В настоящее время отмечается повышенный интерес к метастабильным $\alpha$ - и $\varepsilon$-фазам нового широкозонного полупроводника - оксида галлия $\left(\mathrm{Ga}_{2} \mathrm{O}_{3}\right)$. Достигнут значительный прогресс в выращивании эпитаксиальных пленок [1-9]. Корундообразная $\alpha$-фаза обладает среди всех политипов оксида галлия наибольшей шириной запрещенной зоны $E_{g}$, не менее 5.3 эВ, и при легировании $\mathrm{Sn}$ или $\mathrm{Si}$ имеет высокую донорную проводимость $[3,8,10-15]$. В то же время $\varepsilon$-фаза с $E_{g}$, близкой к 4.9 эВ $[1,2,16]$, является второй после $\beta-\mathrm{Ga}_{2} \mathrm{O}_{3}$ по температурной стабильности. Фаза $\varepsilon-\mathrm{Ga}_{2} \mathrm{O}_{3}$ интересна тем, что обладает спонтанной поляризацией [2]. Оба указанных политипа оксида галлия уже сейчас опробованы для ряда применений $[1,11,17-20]$ в области электронных приборов и сенсоров.

В своей предыдущей работе [20] мы показали возможность разработки селективных датчиков низких концентраций $\mathrm{H}_{2}$ в диапазоне рабочих температур $25-200^{\circ} \mathrm{C}$ на основе структур $\alpha-\mathrm{Ga}_{2} \mathrm{O}_{3} / \mathcal{E}-\mathrm{Ga}_{2} \mathrm{O}_{3}$ с платиновыми контактами. Ключевым фактором для проявления чувствительности структур к водороду $\left(\mathrm{H}_{2}\right)$ является использование каталитически активного металла - платины $(\mathrm{Pt})$ - в качестве электрода. Мы пришли к выводу, что в атмосфере $\mathrm{H}_{2}$ происходит снижение барь- ера Шоттки на границе $\mathrm{Pt} / \alpha-\mathrm{Ga}_{2} \mathrm{O}_{3}$, что соответственно вызывает возрастание тока через структуру. Слои $\alpha-\mathrm{Ga}_{2} \mathrm{O}_{3} / \varepsilon-\mathrm{Ga}_{2} \mathrm{O}_{3}$ с контактами платина-титан $(\mathrm{Pt} / \mathrm{Ti})$, которые также исследовались в ходе этой работы, не показали чувствительности к $\mathrm{H}_{2}$ и другим газам.

Известно, что влажность атмосферы значительно влияет на газовую чувствительность металлооксидных полупроводников. Так, в [21] показано, что в области высоких температур, $T \approx 100-400^{\circ} \mathrm{C}$, имеет место хемосорбция молекул $\mathrm{H}_{2} \mathrm{O}$ на поверхности полупроводников, которая во многих случаях приводит к снижению чувствительности к газам. Это объясняется конкуренцией за центры адсорбции между молекулами $\mathrm{H}_{2} \mathrm{O}$ и молекулами детектируемых газов. Однозначного мнения о механизме хемосорбции молекул $\mathrm{H}_{2} \mathrm{O}$ на поверхности металлооксидных полупроводников нет. При хемосорбции на свободный или занятый кислородом адсорбционный центр молекула $\mathrm{H}_{2} \mathrm{O}$ отдает электроны в зону проводимости полупроводника, в результате чего возрастает электропроводность полупроводников $n$-типа. Многие механизмы хемосорбции молекул $\mathrm{H}_{2} \mathrm{O}$ включают диссоциацию молекулы $\mathrm{H}_{2} \mathrm{O}$ на $\mathrm{H}^{+}$и $\mathrm{OH}^{-}$. Однако энергия диссоциации молекулы $\mathrm{H}_{2} \mathrm{O}$ составляет 4-5эВ, поэтому протекание реакции термостимулированной диссоциации $\mathrm{H}_{2} \mathrm{O}$ в области температур $100-400^{\circ} \mathrm{C}$ на поверхности полупроводника мало ве- 
роятно. Возможна диссоциация молекул $\mathrm{H}_{2} \mathrm{O}$ на поверхности $\beta-\mathrm{Ga}_{2} \mathrm{O}_{3}$ и $\mathrm{GaN}$ при комнатной температуре под действием электромагнитного излучения [22-24]. Кроме этого диссоциация молекул $\mathrm{H}_{2} \mathrm{O}$ на поверхности твердых тел может протекать при локальном увеличении напряженности прикладываемого электрического поля в дефектных областях [21]. При $T \leq 100^{\circ} \mathrm{C}$ на поверхности полупроводников имеет место физическая адсорбция молекул $\mathrm{H}_{2} \mathrm{O}$, которая также может приводить к изменению электрических свойств поверхности.

Детальное исследование влияния влажности атмосферы на характеристики структур $\alpha-\mathrm{Ga}_{2} \mathrm{O}_{3} / \varepsilon-\mathrm{Ga}_{2} \mathrm{O}_{3}$ с контактами из $\mathrm{Pt}$, а также из $\mathrm{Pt} / \mathrm{Ti}$ ранее не проводилось. Однако было показано, что при $T>135^{\circ} \mathrm{C}$ образцы с Рt-контактами не реагировали на воздействие паров $\mathrm{H}_{2} \mathrm{O}$ при относительной влажности атмосферы $\mathrm{RH}=90 \%$. При низких температурах воздействие водяного пара приводило к незначительным изменениям тока, на $1 \%$ для состаренных образцов и 5\% для свежих. Состаренные образцы подвергались длительному (более 6 недель) воздействию $\mathrm{H}_{2}$ в диапазоне концентраций 0.149-1.192 об\% и диапазоне температур $25-200^{\circ} \mathrm{C}$. В наших экспериментах исходно образцы находились в атмосфере чистого сухого воздуха, содержащего не более $10 \mathrm{ppm}$ паров $\mathrm{H}_{2} \mathrm{O}$.

Имеются сведения о том, что влажность атмосферы сказывается на работе различных датчиков на основе $\beta-\mathrm{Ga}_{2} \mathrm{O}_{3}$. Газовые сенсоры резистивного типа на основе тонких пленок и объемного $\beta-\mathrm{Ga}_{2} \mathrm{O}_{3}$ характеризуются рабочими температурами $>400^{\circ} \mathrm{C}$, и их чувствительность слабо подвержена влиянию влажности атмосферы [25]. Однако в области низких температур, $T \leq 150^{\circ} \mathrm{C}$, нанопроволоки $\beta-\mathrm{Ga}_{2} \mathrm{O}_{3}$ также имеют высокую чувствительность к влажности окружающей среды [26,27], воздействие которой приводит к снижению сопротивления нанопроволок. Особенно сильно этот эффект проявляется при $\mathrm{RH} \geq 60 \%$. При $T \leq 50^{\circ} \mathrm{C}$ высокую чувствительность к водяным парам в диапазоне $\mathrm{RH}=11-95 \%$, быстродействие на уровне 10 с и менее показали толстые пленки из наностержней $\beta-\mathrm{Ga}_{2} \mathrm{O}_{3}$ [28]. Отмечается, что эффект влияния влажности атмосферы на характеристики сенсоров усиливается при имплантации $\mathrm{Na}^{+}$и $\mathrm{K}^{+}$в $\beta-\mathrm{Ga}_{2} \mathrm{O}_{3}$. В работе [29] было показано, что возрастание влажности приводит к существенному снижению отклика пленок из нанопроволок $\beta-\mathrm{Ga}_{2} \mathrm{O}_{3}$ на ультрафиолетовое излучение. С учетом возрастающего интереса к метастабильным политипам $\mathrm{Ga}_{2} \mathrm{O}_{3}$ в области разработки газовых, ультрафиолетовых и других типов датчиков существует необходимость исследования влияния влажности на электрическую проводимость структур на основе этого материала.

\section{2. Методика эксперимента}

Эпитаксиальные пленки $\mathrm{Ga}_{2} \mathrm{O}_{3}$ были выращены методом хлоридной газофазной эпитаксии (HVPE) в ООО
„Совершенные кристаллы“. В качестве подложек использовались коммерчески доступные пластины сапфира базисной ориентации (0001) с гладкой и структурированной (PSS) поверхностью толщиной 380-430 мкм. Рельеф PSS представлял собой регулярный массив выступающих правильных конусов, расположенных в гексагональном порядке с шагом 3 мкм. Толщина выращенных слоев на плоской подложке составляла от 1 до 10 мкм, а на PSS - до 4 мкм. Детальное описание процесса изготовления и результаты структурных исследований слоев представлены в работе [3].

Образцы были легированы оловом, концентрация электронов в образцах варьировалась от $10^{17}$ до $5 \cdot 10^{19} \mathrm{~cm}^{-3}$. Для исследования особенностей рельефа поверхности образцов использовался сканирующий электронный микроскоп Tescan MIRA 3 LMU, оснащенный энергодисперсионным рентгеновским спектрометром Oxford Instruments Ultim Мax 40. Сканирование выполнялось при ускоряющем напряжении 20 кВ.

На поверхности структур были сформированы два типа контактов: на основе Pt и Pt/Ti. Металлы наносились методом магнетронного напыления на постоянном токе через маску. При формировании контактов Pt/Ti сначала наносили тонкий слой Ti, затем Pt. Расстояние между электродами составляло 1.85 мм. Толщина контактов $\mathrm{Pt} / \mathrm{Ti}$ составляла $\sim 400 \mathrm{Hм}$, из них толщины слоев Ті и $\mathrm{Pt}$ были равны $\sim 60$ и $\sim 340 \mathrm{Hм} \mathrm{соответственно.}$ Толщина Рt-контактов была 340-360 нм. Все образцы имели одинаковые размеры $2.85 \times 1$ мм.

Таким способом для исследований были сформированы три типа образцов, которые обозначим следующим образом: $\mathrm{Pt} / \alpha-\mathrm{Ga}_{2} \mathrm{O}_{3} / \mathrm{Pt}-$ серия $\mathrm{i} ; \mathrm{Pt} / \alpha-\mathrm{Ga}_{2} \mathrm{O}_{3} /$ $\varepsilon-\mathrm{Ga}_{2} \mathrm{O}_{3} / \mathrm{Pt}$ - серия ii; $\mathrm{Pt} / \mathrm{Ti} / \alpha-\mathrm{Ga}_{2} \mathrm{O}_{3} / \varepsilon-\mathrm{Ga}_{2} \mathrm{O}_{3} / \mathrm{Ti} / \mathrm{Pt}-$ серия iii.

Для исследований влияния влажности на электрические свойства структур использовалась герметичная металлическая камера объемом $950 \mathrm{~cm}^{3}$ со встроенным нагревательным столиком и зондовой установкой. Чистый сухой воздух из генератора подавался на два канала генератора газовых смесей (GMG) „Микрогаз Ф-06“. Генератор чистого воздуха обеспечивал остаточное содержание водяных паров в потоке не более $10 \mathrm{ppm}$. В качестве элементов, регулирующих величину потока в GMG, использовались регуляторы массового расхода газа Bronkhorst El-Flow F-201CV и другие, подобные этой модели. Воздух одного из каналов пропускался через барботер с дистиллированной водой, после чего поступал в гомогенизатор, где смешивался с потоком сухого воздуха из второго канала. При варьировании соотношения потоков через два канала задавался необходимый уровень влажности воздуха в измерительной камере. Суммарный поток через два канала оставался неизменным и составлял $1000 \mathrm{~cm}^{3} /$ мин. В камере для измерения относительной влажности воздуха был расположен датчик емкостного типа НІН 4000 фирмы Honeywell c абсолютной погрешностью $\pm 3.5 \%$, динамическим диапазоном $0-100 \%$ и временем отклика 5 с. 
Согласно техническому описанию, в качестве времени отклика датчика НIH 4000 был выбран временной интервал, за который его показания устанавливаются на уровне $1 / e$ от стационарного значения. При длительной прокачке сухого чистого воздуха через измерительную камеру показания датчика влажности устанавливались на уровне $8-10 \%$. Вероятно, такой уровень RH задается молекулами $\mathrm{H}_{2} \mathrm{O}$, десорбирующимися с поверхности стенок камеры, и среда в камере находится в адсорбционном равновесии. Температура окружающей среды при проведении всех измерений составляла $25^{\circ} \mathrm{C}$.

Измерение временны́х зависимостей сопротивления и вольт-амперных характеристик (BAX) структур при различных условиях эксперимента осуществлялось при помощи источника-измерителя Keithley 2636A. Температура нагрева образцов контролировалась термопарой и регулировалась при помощи программируемого источника постоянного тока DP-832 фирмы Rigol. Процесс измерения сопротивления и ВАX проводился с помощью программы, разработанной в среде Lab View.

\section{3. Структурные исследования}

Фазовый состав эпитаксиальных слоев $\mathrm{Ga}_{2} \mathrm{O}_{3}$ исследовался с помощью рентгено-дифракционного анализа (XRD). На дифрактограммах слоев $\mathrm{Ga}_{2} \mathrm{O}_{3}$ на гладких сапфировых подложках присутствуют только рефлексы при углах $2 \theta=40.25^{\circ}$ и $87.05^{\circ}$, соответствующие отражениям (0006) и $(0.0 .0 .12) \alpha-\mathrm{Ga}_{2} \mathrm{O}_{3}$. Результаты $\omega-2 \theta$-сканирования свидетельствуют о формировании высококачественной эпитаксиальной пленки $\mathrm{Ga}_{2} \mathrm{O}_{3}$ на гладких сапфировых подложках, состоящей из чистой $\alpha$-фазы с ориентацией (0001). Ширина на полувысоте (FWHM) кривой качания наблюдаемых пиков для асимметричного отражения составляла $\sim 10$ угл. мин.

Напротив, образцы, выращенные на профилированных подложках, состояли из смеси $\alpha$ - и $\varepsilon$ - фаз. Кроме дифракционных пиков $\alpha-\mathrm{Ga}_{2} \mathrm{O}_{3}$ наблюдались характерные пики $\varepsilon-\mathrm{Ga}_{2} \mathrm{O}_{3}$ при 19.18, 38.89, 59.90, 83.48 и $112.63^{\circ}$, соответствующие отражениям (0002), (0004), $(0006),(0008)$ и $(0.0 .0 .10)$. Таким образом, эти образцы характеризуются наличием большого количества межфазных границ. Спектры оптического пропускания также подтвердили наличие $\varepsilon$-фазы, которая имеет более узкую оптическую запрещенную зону (4.82 эВ) по сравнению с $\alpha-\mathrm{Ga}_{2} \mathrm{O}_{3}$ (5.06 эВ). Анализ с применением методов сканирующей электронной (SEM) и просвечивающей электронной (ТЕМ) микроскопии показал, что фаза $\alpha-\mathrm{Ga}_{2} \mathrm{O}_{3}$ формирует столбчатые структуры в верхней части конусов, а фаза $\varepsilon-\mathrm{Ga}_{2} \mathrm{O}_{3}$ заполняет промежутки между колоннами. Характерная треугольная огранка структур также указывает на то, что колонны состоят из $\alpha-\mathrm{Ga}_{2} \mathrm{O}_{3}$ с тригональной симметрией. Более глубокий анализ характеристик XRD, SEM и TEM пленок $\mathrm{Ga}_{2} \mathrm{O}_{3}$, выращенных на гладких и профилированных сапфировых подложках, можно найти в работе [3].

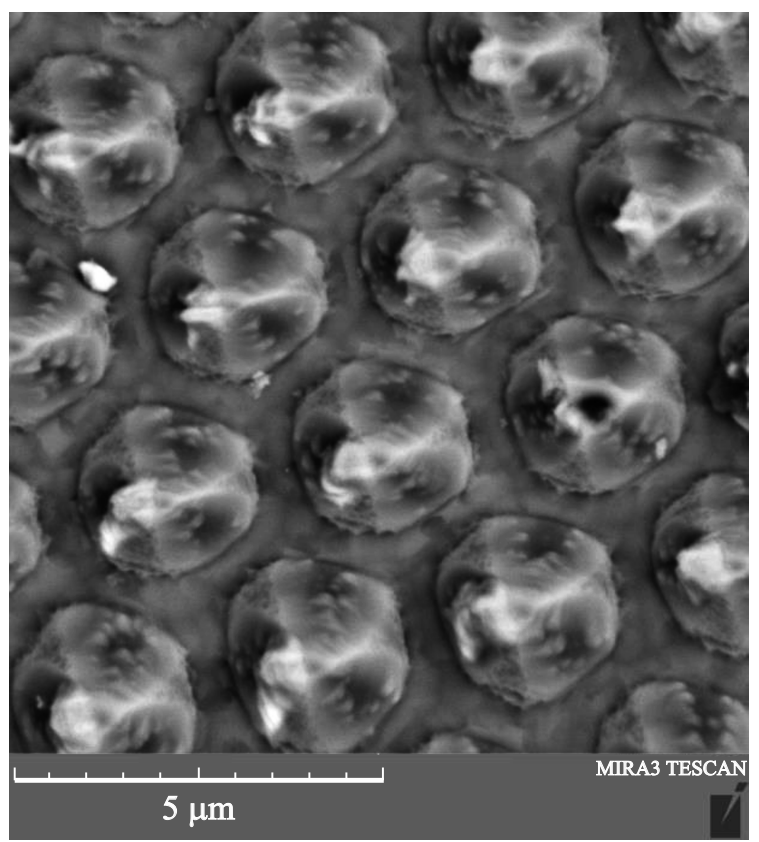

Рис. 1. SEM-изображение поверхности образцов $\alpha-\mathrm{Ga}_{2} \mathrm{O}_{3} /$ $\varepsilon-\mathrm{Ga}_{2} \mathrm{O}_{3}$.

Поверхность образцов, выращенных на подложке PSS, имеет особенности рельефа, которые в зависимости от серии образцов могут различаться по форме. SEM-снимки поверхности образцов серии іiі показаны на рис. 1 . Колонны $\alpha-\mathrm{Ga}_{2} \mathrm{O}_{3}$ на поверхности образцов серии іiі имеют в основании форму неправильного шестиугольника. Энергодисперсионный рентгеновский (EDX) анализ образцов серии іiі показал, что в колоннах $\alpha-\mathrm{Ga}_{2} \mathrm{O}_{3}$ содержится $0.15-0.50$ ат\% хлора, вероятно, проникшего в объем $\alpha-\mathrm{Ga}_{2} \mathrm{O}_{3}$ в процессе синтеза полиморфных структур. Остальные образцы характеризуются отсутствием каких-либо сторонних примесей.

\section{4. Результаты эксперимента и их обсуждение}

На рис. 2 показаны ВАХ образцов при комнатной температуре, $\mathrm{RH}=10$ и $85 \%$. Структуры $\mathrm{Pt} / \alpha-\mathrm{Ga}_{2} \mathrm{O}_{3} / \mathrm{Pt}$ (серия i) на гладких подложках и $\mathrm{Pt} / \mathrm{Ti} / \alpha-\mathrm{Ga}_{2} \mathrm{O}_{3} / \varepsilon-\mathrm{Ga}_{2} \mathrm{O}_{3} / \mathrm{Ti} / \mathrm{Pt}$ (серия iii) на подложках PSS при $\mathrm{RH}=10 \%$ характеризуются низким током, который c увеличением напряжения возрастает по линейному закону. Повышение RH до 85\% в измерительной камере приводит к существенному росту тока в этих образцах. Для серии і ток увеличивается на 2-3 порядка, а для серии іiі - на 4 порядка в диапазоне напряжений $U=5-150$ В. При комнатной температуре и $\mathrm{RH}=85 \%$ ток $I$ образцов серий і и іiі с увеличением напряжения $U$ растет по степенному закону $I \propto U^{m}$ с различными значениями показателя $m: m=(1.44 \pm 0.03)$ для серии і и $m=(0.67 \pm 0.03)$ для серии iii. Наблюдаемая нелиней- 

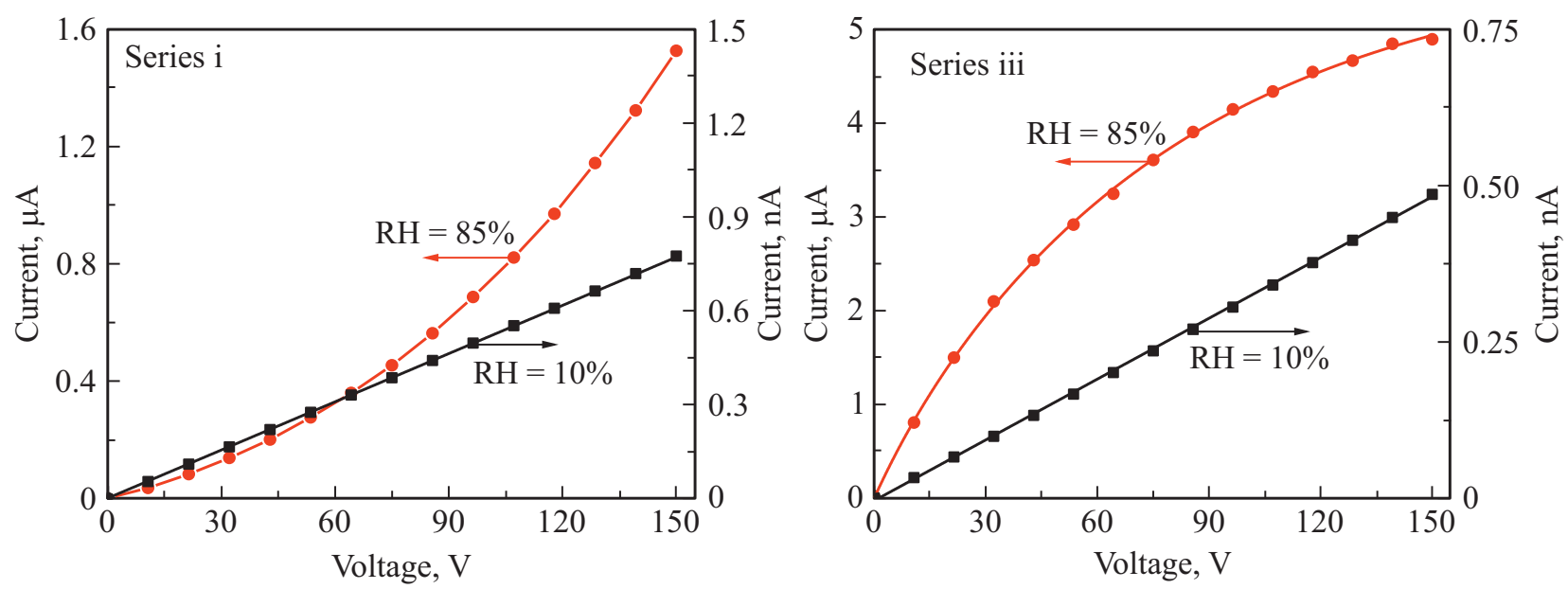

Рис. 2. Вольт-амперные характеристики образцов серий і и іiі при низкой $(10 \%)$ и высокой $(85 \%)$ влажности среды.

Таблица 1. Значения показателя $m$ при $\mathrm{RH}=85 \%$ и разных температурах

\begin{tabular}{c|c|c}
\hline \multirow{2}{*}{$T,{ }^{\circ} \mathrm{C}$} & \multicolumn{2}{|c}{$m$} \\
\cline { 2 - 3 } & $\begin{array}{c}\mathrm{Pt} / \alpha-\mathrm{Ga}_{2} \mathrm{O}_{3} / \mathrm{Pt} \\
\text { (серия i) }\end{array}$ & $\begin{array}{c}\mathrm{Pt} / \mathrm{Ti} / \alpha-\mathrm{Ga}_{2} \mathrm{O}_{3} / \varepsilon-\mathrm{Ga}_{2} \mathrm{O}_{3} / \mathrm{Ti} / \mathrm{Pt} \\
\text { (серия iii) }\end{array}$ \\
\hline 25 & $1.44 \pm 0.03$ & $0.67 \pm 0.03$ \\
50 & $1.16 \pm 0.01$ & $0.77 \pm 0.03$ \\
75 & $1.21 \pm 0.01$ & $0.87 \pm 0.03$ \\
100 & $1.27 \pm 0.01$ & $1.04 \pm 0.03$
\end{tabular}

ность ВАХ при высоком значении RH обусловлена воздействием водяных паров на поверхность исследуемых структур, механизмы которого будут раскрыты далее.

В работе [20] описана чувствительность структур $\mathrm{Pt} / \alpha-\mathrm{Ga}_{2} \mathrm{O}_{3} / \varepsilon-\mathrm{Ga}_{2} \mathrm{O}_{3} / \mathrm{Pt}$ (серия ii) к $\mathrm{H}_{2}$, которая проявляется уже при комнатной температуре. Ток этих образцов при комнатной температуре и $\mathrm{RH}=10 \%$ в диапазоне напряжений $U=5-150 \mathrm{~B}$ составляет единицы мА и значительно превышает ток образцов серий i и iii. Вероятно, помимо разных контактов образцы серии іi характеризуются относительно высокой концентрацией носителей заряда. Экспозиция образцов во влажном воздухе с RH $=85 \%$ практически не ведет к изменению тока. При комнатной температуре и $\mathrm{RH}=10 \%$ ток этих образцов с увеличением напряжения растет по степенному закону $I \propto U^{m}$, где $m=(1.46 \pm 0.02)$. При воздействии $\mathrm{RH}=85 \% m$ практически не изменяется и составляет $(1.48 \pm 0.02)$. При повышении температуры нагрева рассматриваемых образцов до $200^{\circ} \mathrm{C}$ воздействие высокой влажности атмосферы практически не ведет к изменению их тока.

Повышение температуры нагрева образцов серий i и іiі приводит к изменению их реакции на воздействие водяными парами. Показатель степени $m$ для образцов серий і и іiі при $\mathrm{RH}=85 \%$ с повышением температуры изменяется по-разному (см. табл. 1). Однако, судя по отклику сенсоров на воздействие среды при фиксированном значении RH, эффект влияния влажности окружающей среды на электропроводность структур с повышением температуры снижается (рис. 3) и при $T>100^{\circ} \mathrm{C}$ пропадает. В качестве отклика структур $(S)$ на влажность атмосферы было принято следующее отношение:

$$
S=\left(I-I_{10}\right) / I_{10}
$$

где $I$ - ток, текущий через исследуемые образцы при фиксированных значениях $U$ и $\mathrm{RH}, I_{10}$ - ток при $\mathrm{RH}=10 \%$.

Из полученных данных следует, что наибольшим откликом к воздействию парами воды характеризуются структуры $\mathrm{Pt} / \mathrm{Ti} / \alpha-\mathrm{Ga}_{2} \mathrm{O}_{3} / \mathcal{E}-\mathrm{Ga}_{2} \mathrm{O}_{3} / \mathrm{Ti} / \mathrm{Pt}$ (серия iii). Зависимость отклика $S$ этих образцов, определенного по формуле (1), от RH при комнатной температуре

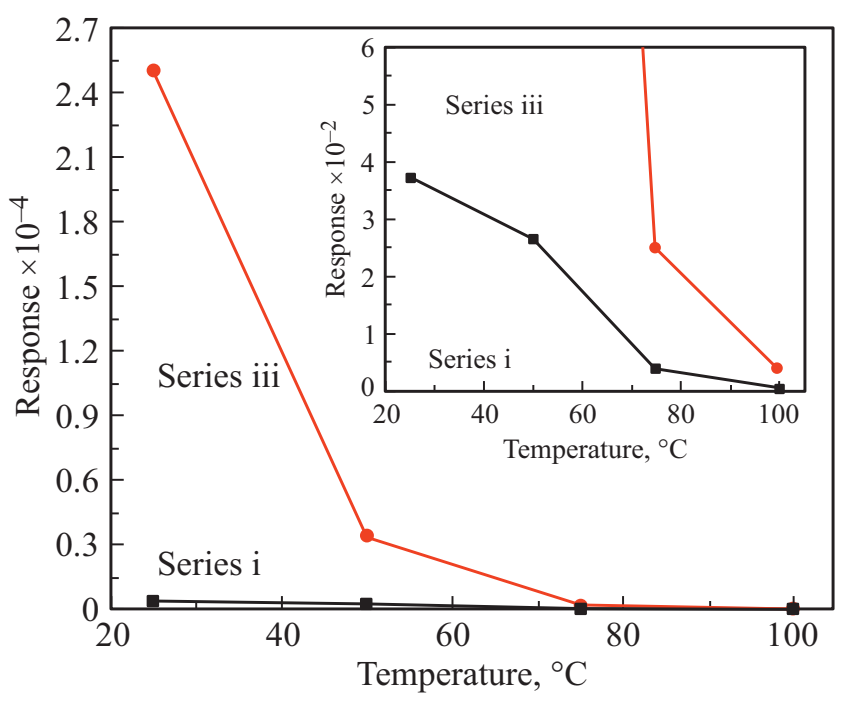

Рис. 3. Температурная зависимость отклика при напряжении 10.7 В для образцов двух серий - i, iii. 


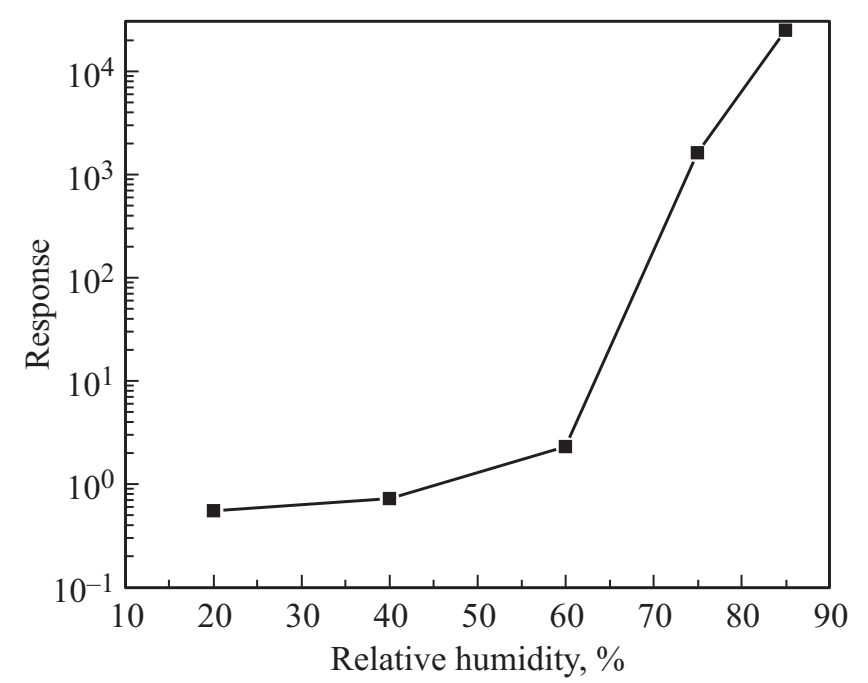

Рис. 4. Зависимость отклика образцов $\mathrm{Pt} / \mathrm{Ti} / \alpha-\mathrm{Ga}_{2} \mathrm{O}_{3} /$ $\varepsilon-\mathrm{Ga}_{2} \mathrm{O}_{3} / \mathrm{Ti} / \mathrm{Pt}$ (серия iii) от RH при $U=10.7 \mathrm{~B}$.

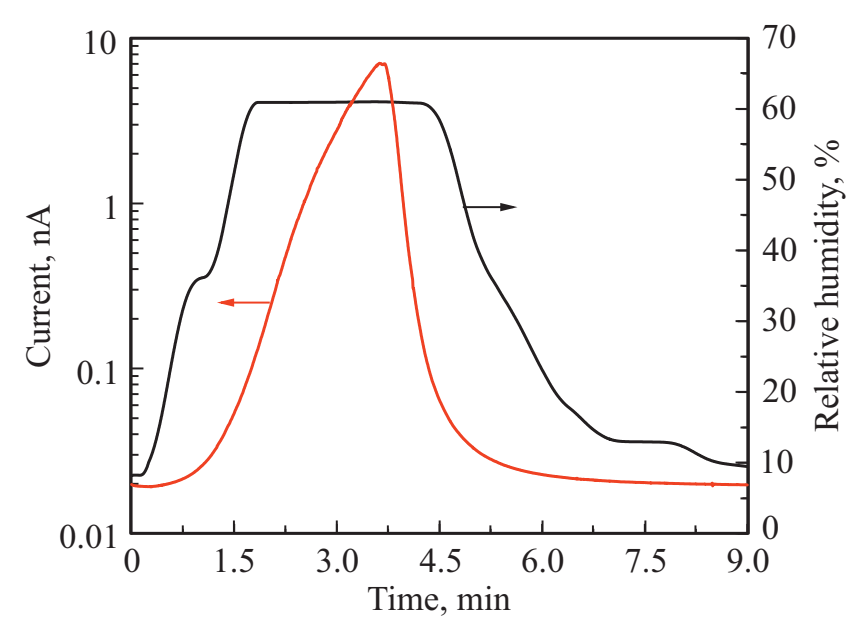

Рис. 5. Временны́е зависимости тока структуры $\mathrm{Pt} / \mathrm{Ti} / \alpha-\mathrm{Ga}_{2} \mathrm{O}_{3} /$ $\varepsilon-\mathrm{Ga}_{2} \mathrm{O}_{3} / \mathrm{Ti} / \mathrm{Pt}$ и уровня $\mathrm{RH}$ в измерительной камере. Квазистационарное значение RH соответствует $61.0 \%$.

показана на рис. 4. При воздействии на образец с $\mathrm{RH} \leq 60 \%$ ток возрастает незначительно, однако при дальнейшем повышении уровня влажности окружающей среды ток резко увеличивается. Из анализа ВАХ при комнатной температуре и разных значениях RH следует, что показатель степени $m$ при снижении RH с 85 до 60\% возрастает с 0.67 до 1.31. При RH $<60 \%$ показатель $m$ близок к единице.

На рис. 5 сопоставлены временнб́е зависимости тока структур $\mathrm{Pt} / \mathrm{Ti} / \alpha-\mathrm{Ga}_{2} \mathrm{O}_{3} / \mathcal{\varepsilon}-\mathrm{Ga}_{2} \mathrm{O}_{3} / \mathrm{Ti} / \mathrm{Pt}$ (серия iii) при напряжении 5В и комнатной температуре, а также уровня RH в измерительной камере. При этом в камере задавался режим $\mathrm{RH}=61.0 \%$. Время установления квазистационарного значения RH в камере объемом $950 \mathrm{~cm}^{3}$ при потоке $1000 \mathrm{~cm}^{3} /$ мин, согласно показаниям датчика НІН 4000, составляло 1-3 мин. Из кривых
Таблица 2. Времена отклика и восстановления структуры $\mathrm{Pt} / \mathrm{Ti} / \alpha-\mathrm{Ga}_{2} \mathrm{O}_{3} / \varepsilon-\mathrm{Ga}_{2} \mathrm{O}_{3} / \mathrm{Ti} / \mathrm{Pt}$ при воздействии различных уровней RH

\begin{tabular}{c|c|c}
\hline RH, \% & $t_{\text {res }}, \mathrm{c}$ & $t_{\text {rec }}, \mathrm{c}$ \\
\hline 36.2 & 450 & 110 \\
61.0 & $>210$ & 145
\end{tabular}

видно, что структуры имеют длительные времена отклика $t_{\text {res }}$, численные значения которых для $\mathrm{RH}=36.2$ и 61.0\% приведены в табл. 2. Однако образцы характеризуются малыми временами восстановления $t_{\text {rec }}$. В качестве $t_{\text {res }}$ был принят промежуток времени между началом действия паров $\mathrm{H}_{2} \mathrm{O}$ на образец и установлением тока на уровне $1.1 I_{\mathrm{RH}}$, где $I_{\mathrm{RH}}-$ стационарное значение тока сенсора при фиксированном значении концентрации водяных паров. В качестве $t_{\text {rec }}$ был принят промежуток времени между началом прокачки сухого воздуха через камеру, в которой расположен образец, и установлением тока образца на уровне $0.9 I_{\mathrm{dry}}$, где $I_{\mathrm{dry}}$ стационарное значение тока образца в сухом воздухе. Полученные значения $t_{\text {res }}$ и $t_{\text {rec }}$ в условиях проведенного эксперимента определяются величиной потока газовой смеси в измерительной камере, объемом измерительной камеры и служат только для оценки быстродействия в выбранных условиях.

Наблюдаемое для структур $\mathrm{Pt} / \alpha-\mathrm{Ga}_{2} \mathrm{O}_{3} / \mathrm{Pt}$ и $\mathrm{Pt} / \mathrm{Ti} / \alpha-\mathrm{Ga}_{2} \mathrm{O}_{3} / \mathcal{E}-\mathrm{Ga}_{2} \mathrm{O}_{3} / \mathrm{Ti} / \mathrm{Pt}$ снижение эффекта влияния влажности окружающей среды с повышением температуры и резкое увеличение тока при $\mathrm{RH} \geq 60 \%$ коррелирует с тем, что наблюдалось в наноструктурах $\beta-\mathrm{Ga}_{2} \mathrm{O}_{3}$ [26-28]. Снижение отклика структур на воздействие водяного пара с повышением температуры свидетельствует о физадсорбции молекул $\mathrm{H}_{2} \mathrm{O}$, которая, как известно [21], наблюдается на поверхности металлооксидных полупроводников при $T \leq 100^{\circ} \mathrm{C}$. На наш взгляд, сильная чувствительность электрических свойств образцов серий і и іiі к воздействию водяными парами может объясняться механизмом Гротгусса (Grotthuss) [21,30,31]. Часть физически адсорбированных молекул $\mathrm{H}_{2} \mathrm{O}$ даже при низких температурах диссоциирует на $\mathrm{H}^{+}$и $\mathrm{OH}^{-}$. Диссоциации способствует высокое приложенное электрическое поле, напряженность которого может существенно возрастать в местах поверхностных дефектов и особенностей рельефа (колонны $\alpha-\mathrm{Ga}_{2} \mathrm{O}_{3}$ ). Согласно механизму Гротгусса, и $\mathrm{H}^{+}$, и $\mathrm{OH}^{-}$обладают подвижностью и могут переносить заряд. При низком уровне RH поверхность образцов слабо покрыта молекулами $\mathrm{H}_{2} \mathrm{O}$ и можно считать, что на поверхности адсорбированы только ионы $\mathrm{OH}^{-}$, которые из-за слабого взаимодействия, имеющего место при физической адсорбции, локализованы в районе центров адсорбции, которыми могут быть поверхностные дефекты. В этом случае носителями заряда являются протоны $\mathrm{H}^{+}$, миграция которых 
Таблица 3. Влияние влажности на характеристики оксидных полупроводников

\begin{tabular}{|c|c|c|c|c|c|}
\hline Материал & $\begin{array}{c}\text { Измеряемая } \\
\text { величина }\end{array}$ & $S$ & $t_{\mathrm{res}}, \mathrm{c}$ & $t_{\mathrm{rec}}, \mathrm{c}$ & Ссылка \\
\hline $\mathrm{Pt} / \mathrm{Ti} / \alpha-\mathrm{Ga}_{2} \mathrm{O}_{3} / \varepsilon-\mathrm{Ga}_{2} \mathrm{O}_{3} / \mathrm{Ti} / \mathrm{Pt}$ & $I$ & $\begin{array}{c}\sim 2.5 \cdot 10^{4} \\
\mathrm{RH}: 10 \rightarrow 85 \%\end{array}$ & $>210$ & 145 & $\begin{array}{l}\text { Данная } \\
\text { работа }\end{array}$ \\
\hline Нанопроволоки $\beta-\mathrm{Ga}_{2} \mathrm{O}_{3}$ & $R$ & $\begin{aligned} \sim 100 & \\
\text { RH: } & 30 \rightarrow 80 \%\end{aligned}$ & 60 & - & {$[27]$} \\
\hline Наностержни $\beta-\mathrm{Ga}_{2} \mathrm{O}_{3}: \mathrm{Na}, \mathrm{K}$ & $Z$ & $\begin{array}{c}\sim 70 \\
\mathrm{RH}: 11 \rightarrow 95 \%\end{array}$ & 6 & 21 & {$[28]$} \\
\hline Нанопроволоки $\beta-\mathrm{Ga}_{2} \mathrm{O}_{3}$ & $I, R$ & $\begin{array}{c}18 \\
\text { RH: } 30 \rightarrow 95 \%\end{array}$ & 18 & 13 & {$[29]$} \\
\hline $\begin{array}{l}\text { Нанокомпозитная пленка } \\
\mathrm{SnO}_{2} / \mathrm{RGO}\end{array}$ & $C$ & $\begin{array}{c}559.85 \\
\text { RH: } 11 \rightarrow 97 \%\end{array}$ & 102 & 6 & {$[30]$} \\
\hline $\begin{array}{l}\text { Композитная тонкая пленка } \\
\mathrm{ZnO} / \mathrm{SnO}_{2}\end{array}$ & $Z$ & RH: $32 \rightarrow 92 \%$ & 17 & 65 & {$[31]$} \\
\hline Нанопроволоки $\mathrm{GeO}^{*}$ & $R$ & $\begin{array}{c}0.21 \\
\text { RH: } 10 \rightarrow 80 \%\end{array}$ & 600 & - & {$[32]$} \\
\hline Тонкая пленка $\mathrm{WO}_{3}$ & $I$ & $\begin{array}{c}0.254 \\
\text { RH: } 42 \rightarrow 95 \%\end{array}$ & 43 & 15 & {$[33]$} \\
\hline
\end{tabular}

Примечание. * Характеристики определены при $T=100^{\circ} \mathrm{C} . R-$ электрическое сопротивление, $Z-$ импеданс, $C-$ электрическая емкость образцов. Запись типа „RH: $10 \rightarrow 85 \%$ “ означает, что в качестве начальной среды для определения отклика был выбран воздух с RH $=10 \%$, а в качестве конечной среды - воздух с $\mathrm{RH}=85 \%$.

протекает посредством прыжков между соседними центрами, занятыми $\mathrm{OH}^{-}$[21]. При высоком уровне RH на поверхности структур между электродами образуются сплошные физически адсорбированные слои. При диссоциации молекулы $\mathrm{H}_{2} \mathrm{O}$ образующийся $\mathrm{H}^{+}$захватывается другой молекулой $\mathrm{H}_{2} \mathrm{O}$, создавая ион $\mathrm{H}_{3} \mathrm{O}^{+}: \mathrm{H}_{2} \mathrm{O}+\mathrm{H}_{2} \mathrm{O} \leftrightarrow \mathrm{H}_{3} \mathrm{O}^{+}+\mathrm{OH}^{-}$. Таким образом, при высокой влажности окружающей среды перенос заряда осуществляется прыжками $\mathrm{H}^{+}$между молекулами $\mathrm{H}_{2} \mathrm{O}$ и ионами $\mathrm{OH}^{-}$. Из этой модели следует, что чем выше RH и больше покрытие поверхности молекулами $\mathrm{H}_{2} \mathrm{O}$, тем больше проводимость по поверхности. При образовании нескольких физически адсорбированных слоев $\mathrm{H}_{2} \mathrm{O}$ на поверхности диссоциация $\mathrm{H}_{2} \mathrm{O}$ резко усиливается [21,30,31].

Из приведенных экспериментальных данных следует, что условием для наблюдения высокой чувствительности к воздействию водяными парами является низкая проводимость материала. На поверхности образцов $\mathrm{Pt} / \alpha-\mathrm{Ga}_{2} \mathrm{O}_{3} / \mathcal{E}-\mathrm{Ga}_{2} \mathrm{O}_{3} / \mathrm{Pt}$ (серия ii) также имеет место формирование физически адсорбированных слоев $\mathrm{H}_{2} \mathrm{O}$, но ток образцов в сухом воздухе настолько велик, что на его фоне эффект влияния влажности окружающей среды несуществен. Образцы $\mathrm{Pt} / \mathrm{Ti} / \alpha-\mathrm{Ga}_{2} \mathrm{O}_{3} / \varepsilon-\mathrm{Ga}_{2} \mathrm{O}_{3} / \mathrm{Ti} / \mathrm{Pt}$ (ceрия іii) отличаются наиболее высокой чувствительностью к воздействию водяными парами в силу большей удельной поверхности из-за особенностей рельефа и наличия дефектных областей на межфазных границах. Повышение температуры способствует переходу от процесса физической адсорбции молекул $\mathrm{H}_{2} \mathrm{O}$ к хемосорб- ции. Хемосорбция характеризуется другой кинетикой, и образование сплошных слоев молекул $\mathrm{H}_{2} \mathrm{O}$ становится затруднительным. В области $T>100^{\circ} \mathrm{C}$, соответствующей хемосорбции молекул $\mathrm{H}_{2} \mathrm{O}$ на поверхности полупроводников, эффект влияния влажности окружающей среды на электрические свойства всех выбранных для исследований образцов не удалось установить.

В табл. 3 приведено сравнение характеристик структур $\mathrm{Pt} / \mathrm{Ti} / \alpha-\mathrm{Ga}_{2} \mathrm{O}_{3} / \mathcal{\varepsilon}-\mathrm{Ga}_{2} \mathrm{O}_{3} / \mathrm{Ti} / \mathrm{Pt}$ (серия iii) при воздействии паров воды с характеристиками наноструктур $\beta-\mathrm{Ga}_{2} \mathrm{O}_{3}$ и других полупроводников при комнатной температуре. Существенная разница в отклике нанопроволок $\beta-\mathrm{Ga}_{2} \mathrm{O}_{3}$ на воздействие водяных паров, вероятно, обусловлена различными параметрами микроморфологии исследуемых образцов. Из таблицы следует, что структуры $\mathrm{Pt} / \mathrm{Ti} / \alpha-\mathrm{Ga}_{2} \mathrm{O}_{3} / \varepsilon-\mathrm{Ga}_{2} \mathrm{O}_{3} / \mathrm{Ti} / \mathrm{Pt}$ не отличаются высоким быстродействием, но характеризуются самым высоким значением отклика. Таким образом, исследованные структуры представляют интерес для разработки на их основе датчиков влажности окружающей среды с низкими рабочими температурами.

\section{5. Заключение}

Исследована зависимость электрической проводимости структур на основе $\alpha-\mathrm{Ga}_{2} \mathrm{O}_{3}$ и $\alpha-\mathrm{Ga}_{2} \mathrm{O}_{3} / \varepsilon-\mathrm{Ga}_{2} \mathrm{O}_{3}$ от уровня влажности окружающей среды в области температур $25-100^{\circ} \mathrm{C}$. Показано, что структуры с платиновыми контактами $\mathrm{Pt} / \alpha-\mathrm{Ga}_{2} \mathrm{O}_{3} / \varepsilon-\mathrm{Ga}_{2} \mathrm{O}_{3} / \mathrm{Pt}$, характеризующиеся высокими током и чувствительностью к 
$\mathrm{H}_{2}$, практически не проявляют реакции на изменение концентрации водяных паров. Структуры $\mathrm{Pt} / \alpha-\mathrm{Ga}_{2} \mathrm{O}_{3} / \mathrm{Pt}$ и $\mathrm{Pt} / \mathrm{Ti} / \alpha-\mathrm{Ga}_{2} \mathrm{O}_{3} / \mathcal{\varepsilon}-\mathrm{Ga}_{2} \mathrm{O}_{3} / \mathrm{Ti} / \mathrm{Pt}$, напротив, характеризующиеся высоким сопротивлением и отсутствием чувствительности к $\mathrm{H}_{2}$, обладают значительной реакцией на изменение уровня влажности атмосферы. Повышение RH c 10 до 85\% при комнатной температуре приводит к росту тока образцов $\mathrm{Pt} / \alpha-\mathrm{Ga}_{2} \mathrm{O}_{3} / \mathrm{Pt}$ на 2-3 порядка, а для образцов $\mathrm{Pt} / \mathrm{Ti} / \alpha-\mathrm{Ga}_{2} \mathrm{O}_{3} / \varepsilon-\mathrm{Ga}_{2} \mathrm{O}_{3} / \mathrm{Ti} / \mathrm{Pt}$ на 4 порядка в диапазоне $U=5-150$ В. Влияние влажности окружающей среды имеет обратимый характер, и наиболее существенные изменения тока образцов наблюдаются при $\mathrm{RH} \geq 60 \%$. С повышением температуры образцов c 25 до $100^{\circ} \mathrm{C}$ эффект влияния водяных паров на электропроводность уменьшается и при $T>100^{\circ} \mathrm{C}$ пропадает. Полученные результаты объясняются в рамках механизма Гротгусса, основанного на проводимости $\mathrm{H}^{+}$ в физически адсорбированных слоях молекул $\mathrm{H}_{2} \mathrm{O}$ и ионов $\mathrm{OH}^{-}$на поверхности твердых тел. Образцы $\mathrm{Pt} / \mathrm{Ti} / \alpha-\mathrm{Ga}_{2} \mathrm{O}_{3} / \varepsilon-\mathrm{Ga}_{2} \mathrm{O}_{3} / \mathrm{Ti} / \mathrm{Pt}$, полученные на подложке PSS, в силу развитого рельефа поверхности и наличия дефектных областей на межфазных границах имеют наибольшую чувствительность к влажности окружающей среды и выглядят перспективными для создания сенсоров уровня влажности RH в атмосфере с низкими рабочими температурами.

\section{Благодарности}

SEM- и EDX-исследования образцов серии iii проведены с использованием оборудования центра коллективного пользования „Аналитический центр геохимии природных систем“ НИ ТГУ.

\section{Финансирование работ}

Исследование выполнено при поддержке гранта Российского научного фонда (проект № 20-79-10043).

\section{Конфликт интересов}

Авторы заявляют, что у них нет конфликта интересов.

\section{Список литературы}

[1] M. Pavesi, F. Fabbri, F. Boschi, G. Piacentini, A. Baraldi, M. Bosi, E. Gombia, A. Parisini, R. Fornari. Mater. Chem. Phys., 205, 502 (2018).

[2] F. Mezzadri, G. Calestani, F. Boschi, D. Delmonte, M. Bosi, R. Fornari. Inorg. Chem., 55 (22), 12079 (2016).

[3] S. Shapenkov, O. Vyvenko, E. Ubyivovk, O. Medvedev, G. Varygin, A. Chikiryaka, A. Pechnikov, M. Scheglov, S. Stepanov, V. Nikolaev. Phys. Status Solidi A, 217, 1900892 (2020).

[4] T. Ma, X. Chen, F. Ren, S. Zhu, S. Gu, R. Zhang, Y. Zheng, J. Ye. Semiconductots, 40, 012804 (2019).
[5] H. Sun, K.H. Li, C.G.T. Castanedo, S. Okur, G.S. Tompa, T. Salagaj, S. Lopatin, A. Genovese, X. Li. Cryst. Growth Des., 18, 2370 (2018).

[6] Y. Oshima, E.G. Víllora, K. Shimamura. Appl. Phys. Express, 8, 055501 (2015).

[7] Y. Yao, L.A.M. Lyle, J.A. Rokholt, S. Okur, G.S. Tompa, T. Salagaj, N. Sbrockey, R.F. Davis, L.M. Porter. ECS Trans., 80 (7), 191 (2017).

[8] V.I. Nikolaev, S.I. Stepanov, A.I. Pechnikov, S.V. Shapenkov, M.P. Scheglov, A.V. Chikiryaka, O.F. Vyvenko. ECS J. Solid State Sci. Technol., 9, 045014 (2020).

[9] А.И. Печников, С.И. Степанов, А.В. Чикиряка, М.П. Щеглов, М.А. Одноблюдов, В.И. Николаев. ФТП, 6, 789 (2019).

[10] S.I. Stepanov, V.I. Nikolaev, V.E. Bougrov, A.E. Romanov. Rev. Adv. Mater. Sci., 44, 63 (2016).

[11] E. Ahmadi, Y. Oshima. J. Appl. Phys., 126, 160901 (2019).

[12] В.И. Николаев, А.И. Печников, Л.И. Гузилова, А.В. Чикиряка, М.П. Щеглов, В.В. Николаев, С.И. Степанов, А.А. Васильев, И.В. Щемеров, А.Я. Поляков. Письма ЖТФ, 46 (5), 27 (2020).

[13] A.Y. Polyakov, V.I. Nikolaev, S.I. Stepanov, A.I. Pechnikov, E.B. Yakimov, N.B. Smirnov, I.V. Shchemerov, A.A. Vasilev, A.I. Kochkova, A.V. Chernykh, S.J. Pearton. ECS J. Solid State Sci. Technol., 9, 045003 (2020).

[14] A.Y. Polyakov, N.B. Smirnov, I.V. Shchemerov, E.B. Yakimov, V.I. Nikolaev, S.I. Stepanov, A.I. Pechnikov, A.V. Chernykh, K.D. Shcherbachev, A.S. Shikoh, A.I. Kochkova, A.A. Vasilev, S.J. Pearton. APL Mater., 7, 051103 (2019).

[15] A. Parisini , A. Bosio, V. Montedoro, A. Gorreri, A. Lamperti, M. Bosi, G. Garulli, S. Vantaggio, R. Fornari. APL Mater., 7, 031114 (2019).

[16] M. Mulazzi, F. Reichmann, A. Becker, W. M. Klesse, P. Alippi, V. Fiorentini, A. Parisini, M. Bosi, R. Fornari. APL Mater., 7, 022522 (2019).

[17] Y.M. Lu, C. Li, X.H. Chen, S. Han, P.J. Cao, F. Jia, Y.X. Zeng, X.K. Liu W.Y. Xu, W.J. Liu, D.L. Zhu. Chinese Physics B, 28, 018504 (2019).

[18] Y. Arata, H. Nishinaka, D. Tahara, M. Yoshimoto. Jpn. J. Appl. Phys., 59 (2), (2017).

[19] В.М. Калыгина, В.И. Николаев, А.В. Алмаев, А.В. Цымбалов, В.В. Копьев, Ю.С. Петрова, И.А. Печников, П.Н. Бутенко. ФТП, 10, 1035 (2020).

[20] A.V. Almaev, V.I. Nikolaev, S.I. Stepanov, A.I. Pechnikov, A.V. Chikiryaka, N.N. Yakovlev, V.M. Kalygina, V.V. Kopyev, E.V. Chernikov. J. Phys. D: Appl. Phys., 53, 414004 (2020).

[21] H. Farahani, R. Wagiran, M.N. Hamidon. Sensors, 14, 7881 (2014).

[22] S.J. Chang, Y.L. Wu, W.Y. Weng, Y.-H. Lin, W.K. Hsieh, J.K. Sheu, C.L. Hsu. J. Electrochem. Soc., 161 (9), H508 (2014).

[23] J.S. Hwang, T.Y. Liu, S. Chattopadhyay, G.M. Hsu, A.M. Basilio, H.W. Chen, Y.K. Hsu, W.H. Tu, Y.-G. Lin, K.H. Chen, C.-C. Li, S.B.Wang, H.Y. Chen, L.C. Chen. Nanotechnology, 24, 055401 (2013).

[24] W. He, W. Wu, Q. Li, K. Chen, X. Lu. Chem. Nano Mater., 6, 208 (2020).

[25] A. Afzal. J. Materiomics, 5, 542 (2019).

[26] G. Domènech-Gil, I.P. Riera, E. López-Aymerich, P. Pellegrino, S. Barth, I. Gràcia, C. Cané, J.D. Prades, M. MorenoSereno, A. Romano-Rodriguez. Proceedings, 1(4), 468 (2017). https://doi.org/10.3390/proceedings 1040468 
[27] G. Domenech-Gil, I. Riera, E. Lopez-Aymerich, M. Moreno, P. Pellegrino, I. Gracia, C. Cane, S. Barth, A. Romano-Rodriguez. Proceedings, 2 (13), 958 (2018). https://doi.org/10.3390/proceedings2130958

[28] D. Wang, Y. Lou, R. Wang, P. Wang, X. Zheng, Y. Zhang, N. Jiang. Ceram. Int., 41, 14790 (2015).

[29] Y.M. Juan, S.J. Chang, H.T. Hsueh, S.H. Wang, W.Y. Weng, T.C. Cheng, C.L. Wu. RSC Adv., 5, 84776 (2015).

[30] D. Zhang, H. Chang, P. Li, R. Liu, Q. Xue. Sensors Actuators B, 225, 233 (2016).

[31] M. Velumani, S.R. Meher, Z.C. Alex. Sensors Actuators B, 301, 127084 (2019).

[32] J. Samá, M.S. Seifner, G. Doménech-Gil, J.K. Santander. Sensors Actuators B, 243, 669 (2017).

[33] Y.M. Juan, S.J. Chang, H.T. Hsueh, T.C. Chen, S.W. Huang, Y.H. Lee, T.J. Hsueh, C.L. Wu. Sensors Actuators B, 219, 43 (2015).

Редактор Л.В. Шаронова

\title{
Effect of ambient humidity on the electrical conductivity properties of polymorphic $\mathrm{Ga}_{2} \mathrm{O}_{3}$ structures
}

\author{
A.V. Almaev1, V.I. Nikolaev ${ }^{2,3}$, S.I. Stepanov ${ }^{\mathbf{2} 3}$, \\ N.N. Yakovlev ${ }^{1}$, A.l. Pechnikov ${ }^{3}$, E.V. Chernikov', \\ B.O. Kushnarev ${ }^{1}$ \\ ${ }^{1}$ National Research Tomsk State University, \\ 634050 Tomsk, Russia \\ 2 Perfect Crystals LLC, \\ 194064 St. Petersburg, Russia \\ 3 loffe Institute, \\ 194021 St. Petersburg, Russia
}

\begin{abstract}
The effect of ambient humidity on the electrical conductivity properties of $\alpha \mathrm{Ga}_{2} \mathrm{O}_{3}$ and $\alpha-\mathrm{Ga}_{2} \mathrm{O}_{3} / \varepsilon-\mathrm{Ga}_{2} \mathrm{O}_{3}$ structures has been studied. Polymorphic $\mathrm{Ga}_{2} \mathrm{O}_{3}$ epitaxial layers were deposited by chloride vapor phase epitaxy on sapphire substrates. $\mathrm{Pt}$ and $\mathrm{Pt} / \mathrm{Ti}$ were used as contacts. It was found that $\mathrm{Pt} / \alpha-\mathrm{Ga}_{2} \mathrm{O}_{3} / \mathrm{Pt}$ and $\mathrm{Pt} / \mathrm{Ti} / \alpha-\mathrm{Ga}_{2} \mathrm{O}_{3} / \varepsilon-\mathrm{Ga}_{2} \mathrm{O}_{3} / \mathrm{Ti} / \mathrm{Pt}$ structures exhibited a high sensitivity of the current-voltage characteristics to atmospheric humidity in the temperature range $25-100^{\circ} \mathrm{C}$. It was found that the effect of water vapor on the currentvoltage characteristics of the structures was reversible and the most significant changes in the current in the samples were observed at a relative humidity $\mathrm{RH} \geq 60 \%$. With increasing temperature the effect of atmospheric humidity on the current? voltage characteristics decreased and disappeared at $T>100^{\circ} \mathrm{C}$. The experimental results obtained are explained in terms of the Grottguss mechanism.
\end{abstract}

